\title{
Macronuclear Transformation with Specific DNA Fragments Controls the Content of the New Macronuclear Genome in Paramecium tetraurelia $\dagger$
}

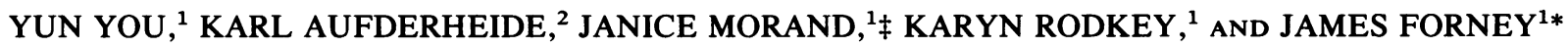 \\ Department of Biochemistry, Purdue University, West Lafayette, Indiana 47907, ${ }^{1}$ and \\ Department of Biology, Texas A\&M University, College Station, Texas $77843^{2}$
}

Received 2 July 1990/Accepted 26 November 1990

\begin{abstract}
A previously isolated mutant cell line called d48 contains a complete copy of the A surface antigen gene in the micronuclear genome, but the gene is not incorporated into the macronucleus. Previous experiments have shown that a cytoplasmic factor made in the wild-type macronucleus can rescue the mutant. Recently, $S$. Koizumi and S. Kobayashi (Mol. Cell. Biol. 9:4398-4401, 1989) observed that injection of a plasmid containing the $A$ gene into the $\mathbf{d} 48$ macronucleus rescued the cell line after autogamy. It is shown here that an 8.8-kb EcoRI fragment containing only a portion of the A gene coding region is sufficient for the rescue of d48. The inability of other A gene fragments to rescue the mutant shows that this effect is dependent upon specific Paramecium DNA sequences. Rescue results in restoration of the wild-type DNA restriction pattern in the macronucleus. These results are consistent with a model in which the macronuclear $A$ locus normally makes an additional gene product that is required for correct processing of the micronuclear copy of the $\mathbf{A}$ gene.
\end{abstract}

Paramecium tetraurelia possesses two distinctly differentiated types of nuclei. The polygenomic macronucleus is transcriptionally active and determines the phenotype of the vegetative cell. The diploid micronuclei (two per cell) are transcriptionally inactive but participate in the nuclear events of sexual reorganization (i.e., conjugation [a reciprocal sexual exchange with another cell of the complementary mating type] and autogamy [self-fertilization]). Although the two types of nuclei in $P$. tetraurelia are mitotic products from a single fertilization nucleus, the dramatic molecular rearrangements associated with nuclear differentiation provide the opportunity for differences in genetic content between the macronucleus and the micronucleus. Sonneborn and Schneller (18) documented a number of examples in which differences between macronuclear expression and apparent micronuclear genetic content are characterized by non-Mendelian inheritance of specific traits.

Propagation of a macronuclear differentiated phenotype through multiple cycles of sexual reorganization is well illustrated by the $\mathrm{d} 48$ cell line. Originally isolated after X-ray mutagenesis, it is unable to express the serotype $A$ antigen gene under conditions that usually induce expression. The $\mathbf{A}$ surface protein is one member of a family of variable surface proteins in paramecia (reviewed in references 1 and 14). Southern blots of d48 DNA indicate that most of the A gene coding region is either not present in the macronucleus or present at an extremely low copy number. However, genetic and molecular evidence indicates that the $\mathrm{d} 48$ cell line contains a complete copy of the $\mathbf{A}$ gene in its micronucleus $(3,13)$. The striking feature of this mutant is the influence that the cytoplasm exerts during the formation of the new macronucleus. Unlike Mendelian mutations which segregate in the $F_{2}$ generation, the $A^{-}$phenotype of $d 48$ is inherited

\footnotetext{
* Corresponding author.

$\dagger$ Journal paper 12657 from the Purdue University Agricultural Experiment Station.

$\ddagger$ Present address: Department of Biochemistry and Biophysics, University of California at Davis, Davis, CA 95616.
}

according to the cytoplasm of the parental cell; typically, mutant cells are derived from the $\mathrm{d} 48$ parent and wild-type progeny are derived from the wild-type parent. The cytoplasm of the wild type is dominant; that is, a mutant cell is never derived from a parent with wild-type cytoplasm but occasionally wild-type progeny are produced from a mutant parental cell (3). Transfer of macronucleoplasm from the wild type to d48 has shown that the old macronucleus is the source of the cytoplasmic determinant (7). More recently, it has been shown that cytoplasmic transfers from wild-type cells to $\mathrm{d} 48$ cells rescue the mutant if recipient and donor cells are both at the appropriate stage of macronuclear development (8). Together, these experiments indicate that the d48 macronucleus fails to produce a factor that is normally required for correct processing of the $A$ gene.

The non-Mendelian inheritance of $\mathrm{d} 48$ is characteristic of other traits in paramecia, such as the mating type and an induced mutation affecting trichocyst discharge (18); therefore, this pattern of inheritance is not isolated to the $A$ surface antigen locus. The paradox posed by this mutation is its specificity despite its action through the cytoplasm. An attractive model could be constructed if each genetic locus produced a product necessary for its own processing in the next macronucleus. The recent development of protocols for transformation of paramecia using cloned genes permits determination of whether or not the A gene itself is capable of establishing the wild-type cytoplasmic state necessary for incorporation of the A gene in the newly developing macronucleus. A preliminary report by Koizumi and Kobayashi (8), as well as unpublished work from our own laboratories and other independent workers (11), has indicated that microinjection of $\mathrm{d} 48$ cells with a plasmid containing a cloned serotype A gene was sufficient to rescue the mutant after nuclear reorganization.

In this report, we show that this is a specific effect of the A gene sequences, that the wild-type macronuclear DNA restriction pattern is regenerated in the rescued cell lines, and that a fragment of the A gene, which is unable to express the A surface protein, is nevertheless sufficient to rescue the 
mutant. These results are consistent with a model in which the A gene produces an additional product unrelated to its known function as a surface protein. This product is made in the macronucleus; is transported to the cytoplasm, where it interacts with the developing macronucleus; and is necessary for correct processing of the A gene. These results are discussed in relation to other traits in paramecia determined by stable macronuclear differentiation.

\section{MATERIALS AND METHODS}

Cell lines and cultivation. Homozygous strain d48 $t w$ (twisty) of $P$. tetraurelia (derived from stock 51) was obtained from the laboratory of John R. Preer (Indiana University, Bloomington). Cells were cultured either in (i) $0.25 \%$ Wheat Grass medium (Pines International, Lawrence, Kans.) buffered with sodium phosphate or (ii) $0.15 \%$ Baked Lettuce medium buffered with excess $\mathrm{CaCO}_{3}$. In some cases, the medium was augmented with $5 \mathrm{mg}$ of stigmasterol (Sigma) per liter. Before use, the sterile medium was inoculated with a nonpathogenic strain of Klebsiella pneumoniae (ATCC 27889) as a food organism. Basic methods of culture, induction and assessment of autogamy, harvesting of cells, and detection of surface antigens, etc., have been described by Sonneborn $(15,16)$.

Microinjection. Microinjections were performed as previously described in Godiska et al. (6). Briefly, DNA was dissolved at a concentration of $1 \mathrm{mg} / \mathrm{ml}$ (J. Forney laboratory) or $5 \mathrm{mg} / \mathrm{ml}$ (K. Aufderheide laboratory) in buffer specified by Tondravi and Yao (20). Approximately 3 to $6 \mathrm{pl}$ of the solution was injected into the macronuclei of selected recipient cells. The glass microneedles used were 1 to $2 \mu \mathrm{m}$ in diameter at the tips. After injection, cells were returned to fresh medium as described below. Survival rates of 80 to $90 \%$ of the injected cells were routinely observed with this protocol.

Cloned DNA. Plasmid pSA14SB (6) was obtained from John R. Preer. Plasmid pSA10XS was constructed by digesting $\lambda$ SA1 (4) with $S a l I$ and $X h o I$ and ligating the $10-\mathrm{kb}$ fragment into the SalI site of pUC119 (21). pSA8.8R and pSA2.1H contain EcoRI and HindIII fragments ligated into the EcoRI and HindIII sites of pUC119.

Preparation of paramecium DNA. Approximately $100-\mathrm{ml}$ cultures of paramecia ( 150,000 cells) were resuspended in 0.4 $\mathrm{ml}$ of their own culture fluid and quickly added to $0.8 \mathrm{ml}$ of lysing solution (1\% sodium dodecyl sulfate- 0.05 or $0.5 \mathrm{M}$ disodium EDTA-100 mM Tris hydrochloride ( $\mathrm{pH} \mathrm{9.5)} \mathrm{at}$ $65^{\circ} \mathrm{C}$. DNA was prepared from lysates by two rounds of phenol-chloroform extraction and ethanol precipitation.

Southern blots and hybridizations. Southern transfers onto Nytran or nitrocellulose filters (Schleicher \& Schuell Inc., Keene, N.H.) were performed as described by Maniatis et al. (10). Before hybridization, filters were prewashed with $10 \times$ Denhardt solution- $-0.1 \%$ sodium dodecyl sulfate- $0.2 \mathrm{M}$ phosphate buffer- $5 \times$ SET $(1 \times$ SET is $0.15 \mathrm{M} \mathrm{NaCl}, 0.03 \mathrm{M}$ Tris, and $2 \mathrm{mM}$ EDTA) at $68^{\circ} \mathrm{C}$ for $1 \mathrm{~h}$. The filters were prehybridized with $5 \times$ SET- $0.2 \mathrm{M}$ phosphate buffer-1 $\times$ Denhardt solution- $0.5 \%$ sodium dodecyl sulfate at $68^{\circ} \mathrm{C}$. After $1 \mathrm{~h}$, labeled probe was added. After 12 to $15 \mathrm{~h}$, the filters were washed in $0.2 \times \mathrm{SET}-0.025 \mathrm{M}$ phosphate buffer$0.1 \%$ sodium $P P_{i}-0.1 \%$ sodium dodecyl sulfate three times for 30 min each time at $68^{\circ} \mathrm{C}$. The filters were air dried and exposed to Kodak X-Omat $\mathrm{AR}$ film at $-70^{\circ} \mathrm{C}$ with Cronex Lightning-Plus intensifier screens.

Sexual reproduction in paramecia. Vegetative cell cultures must periodically undergo sexual reproduction to avoid senescence and eventual death. Both conjugation and a self-fertilization event called autogamy involve a similar series of nuclear divisions. The nuclear events of autogamy are outlined in the following description (reviewed in reference 17). Once a cell culture has undergone at least 20 fissions since the last nuclear reorganization event, starvation induces autogamy. The two diploid micronuclei undergo meiosis, leading to production of eight haploid products. All but one of these products are destroyed. The remaining haploid nucleus divides mitotically, forming two haploid products which fuse to create the single fertilization nucleus. Two mitotic divisions result in four diploid products, of which two become new macronuclei and two become micronuclei. Within the same cell, the old macronucleus begins to break into smaller fragments that are transcriptionally active but no longer able to replicate their DNA. Thus, formation of new macronuclei occurs in the backdrop of the still-functional old macronucleus.

Scoring for rescue of $\mathrm{d} 48$. Cells of the $\mathrm{d} 48$ variant were brought through autogamy and cultured at room temperature for approximately 5 to $\mathbf{1 0}$ generations. Before injection, the cells were routinely checked for serotype A by using anti-A serum (Indiana University collection). Any cell lines expressing A represent spontaneous revertants and were discarded. Selected cells were injected as described above, placed into individual depression wells, and cultured for 2 days at $34^{\circ} \mathrm{C}$ to induce expression of serotype A. After scoring for A expression, the cell lines were shifted to $27^{\circ} \mathrm{C}$ and subcultured until they were at least 20 generations from injection. After induction of autogamy by starvation, autogamous cells were isolated into separate cultures and kept for 2 days at $27^{\circ} \mathrm{C}$, followed by induction of $\mathrm{A}$ expression at $34^{\circ} \mathrm{C}$. Lines able to express $\mathrm{A}$ after this treatment were considered to have been rescued to the wild type.

\section{RESULTS}

Rescue by plasmids containing the entire $A$ gene. A brief description of the nuclear events associated with sexual reproduction in paramecia is provided in Materials and Methods. Since the phenotype and genotype of cells both before and after nuclear reorganization will be presented, the terminology throughout the text will refer to transformed cell lines as lines before autogamy that contain the injected plasmid DNA, and rescued cell lines refer to cells after formation of a new macronucleus that contain a functional copy of the A gene in the macronucleus.

In the initial report that a cloned copy of the A gene could rescue the d48 mutant, plasmid pSA14SB (8) was injected into the macronucleus of $\mathrm{d} 48$. A map of the A surface antigen gene locus and the relative location of the pSA14SB insert is shown in Fig. 1. The pSA14SB insert contains the entire A antigen gene coding region as well as flanking sequences on the $5^{\prime}$ and $3^{\prime}$ ends. We confirmed that this plasmid could rescue cell lines that were stably transformed by microinjection (Table 1). Stable transformants are defined as cell lines that express the A surface antigen just before autogamy or contain the injected DNA immediately before autogamy. For plasmids that contain the entire $A$ gene, expression of $A$ surface protein was always correlated with the presence of the plasmid DNA. Injections performed in the laboratories of both K. Aufderheide and J. Forney gave similar results, although there was some variation in the frequency of rescue. This difference could be the result of subtle variations in culture conditions or the consequence of differences between the $\mathrm{d} 48$ stock cultures used for injection. Once 


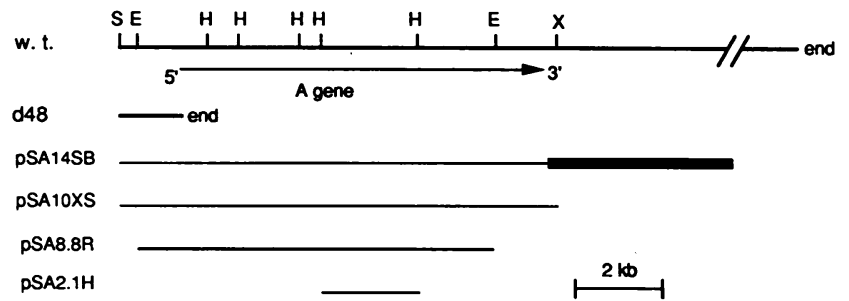

FIG. 1. Map of the A surface antigen gene region. The wild-type (w.t.) and $\mathbf{4} 48$ macronuclear DNA fragments are shown, and the A gene transcript is indicated by the arrow. The relative locations of DNA fragments cloned into recombinant plasmids and used in this study are indicated below the map. The black box on pSA14SB indicates Paramecium DNA sequences not found at the A locus (see text). E, EcoRI; H, HindIII; S, SalI; X, XhoI.

rescued, these cell lines were permanently wild type and never reverted to the mutant phenotype, even after multiple rounds of autogamy (data not shown).

pSA14SB contains approximately $3.5 \mathrm{~kb}$ of DNA 3 ' to the $A$ gene that is not found at the native macronuclear $A$ gene locus and is presumably a cloning artifact (Fig. 1). To determine whether these non-A gene sequences were responsible for rescue of the d48 cell line, a SalI-XhoI fragment from $\lambda \mathrm{SA}-1$ (4) was cloned into the SalI site of pUC119. This clone, pSA10XS, was used to transform d48 cells. pSA10XS was also sufficient to rescue the mutant (Table 1); in fact, $100 \%$ of the stable transformants were rescued after formation of a new macronucleus. To determine whether the wild-type DNA restriction pattern was present in the rescued d48 cell lines, DNAs were isolated from d48 cell lines transformed with pSA10XS and the rescued cell line derived from that transformant. Southern blots of the HindIII- and XhoI-digested DNA were probed with pSA10XS and compared with wild-type DNA and pSA10XS. The transformed cells contained the pattern expected for the plasmid, and rescued cells had the wild-type restriction pattern (Fig. 2). The differences between the wild-type pattern and the expected plasmid bands (Fig. 2, compare lane 1 with lanes 3 and 5) are the absence of a $6.2-\mathrm{kb}$ band and the presence of a $3.8-\mathrm{kb}$ band in the wild-type genome. The $6.2-\mathrm{kb}$ band present in the HindIII$X$ hol digest of pSA10XS is the result of the absence of the $X h o I$ site in the plasmid. All of the rescued cell lines that were examined contained the wild-type pattern.

Rescue of $\mathrm{d} 48$ with a fragment unable to express the $A$ surface protein. The A surface antigen is the only known coding function of the DNA sequences present in the pSA10XS clone. To determine whether the entire A gene is necessary to rescue the d48 mutant cell line, a subclone containing an 8.8-kb EcoRI fragment (pSA8.8R) was used to transform $\mathrm{d} 48$ cells. Unlike the previously described plas-

TABLE 1. Efficiency of rescue by macronuclear transformation

\begin{tabular}{lccc}
\hline $\begin{array}{c}\text { DNA } \\
\text { injected }\end{array}$ & $\begin{array}{c}\text { No. of stable } \\
\text { transformed } \\
\text { lines }\end{array}$ & $\begin{array}{c}\text { No. of rescued lines } \\
\left(\mathrm{A}^{+} \text {after autogamy) }\right.\end{array}$ & $\begin{array}{c}\text { \% of transformed } \\
\text { lines that were } \\
\text { rescued }\end{array}$ \\
\hline pSA14SB & 10,117 & 10,102 & 100,87 \\
pSA10XS & 13 & 13 & 100 \\
pSA8.8R & 14 & 14 & 100 \\
pSA2.1H & 12 & 0 & 0 \\
\hline
\end{tabular}

${ }^{a}$ Data obtained from different laboratories are shown separately.

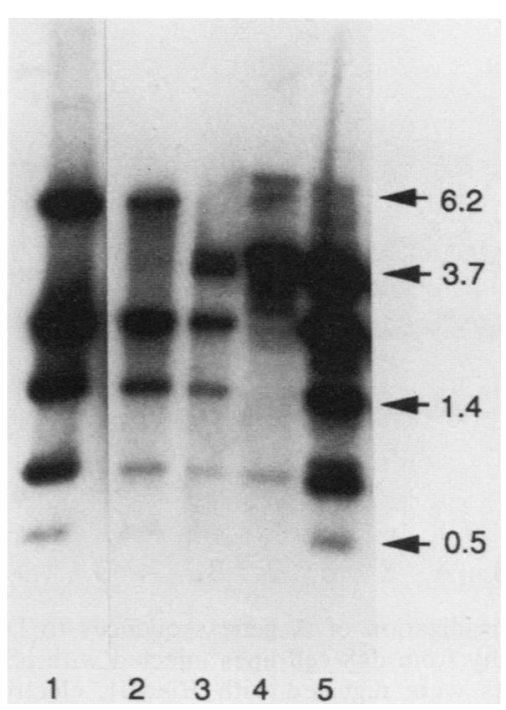

FIG. 2. Hybridization of A gene sequences to transformed and rescued d48 cell lines. Total genomic DNA (approximately $5 \mu \mathrm{g}$ ) was digested with HindIII and $X h o I$ restriction enzymes, electrophoresed on $0.8 \%$ agarose, blotted to nitrocellulose, and hybridized with ${ }^{32} \mathrm{P}$-labeled pSA10XS. Size markers are indicated in kilobases. Lanes: 1, pSA10XS; 2 d48 cell line transformed with pSA10XS; 3 , rescued d48 cell line (after autogamy); 4, d48; 5, wild type. Exposure time for the autoradiogram was $24 \mathrm{~h}$, except for lane 2 , which was exposed for only $4 \mathrm{~h}$ because of the high copy number present in the transformed cells.

mids, this subclone does not contain the entire coding region of the A gene and cannot express the A antigen on the cell surface. Since the only marker for transformation currently available is A surface protein expression, it was not possible to determine which of the injected cell lines were transformed unless hybridization analysis was performed on DNA samples from individual cell lines. To avoid DNA analysis of every injected cell line, a portion of each culture was kept at $13^{\circ} \mathrm{C}$ and fed one fission per week; this slow growth prevents the cell line from reaching the number of cell divisions required for induction of autogamy. The remaining cells were cultured for greater than 20 fissions, starved to induce autogamy, and then scored for $\mathbf{A}$ antigen expression. The cultures kept at $13^{\circ} \mathrm{C}$, corresponding to rescued cell lines after autogamy, were placed at $27^{\circ} \mathrm{C}$ and allowed to multiply, and DNA was isolated. As a control, DNA isolated from an equal number of nonrescued cell lines before autogamy was analyzed. There was complete correlation between rescue after formation of a new macronucleus and transformation with pSA8.8R (Table 1). Figure 3 shows a representative example of DNA isolated from six cell lines before autogamy; lanes 3 to 5 contained DNAs from cell lines that were rescued after autogamy, and lanes 6 to 8 contained DNAs from control cell lines that were not rescued. None of the control cells (injected with pSA8.8R but not rescued) were transformed. Northern (RNA) analysis of total RNAs isolated from cell lines transformed with pSA8.8R indicated that there was no detectable steady-state level of truncated A gene mRNA in these cells (data not shown). These results demonstrated that the 8.8-kb EcoRI fragment was sufficient to rescue $\mathrm{d} 48$ although it could not express normal levels of the A surface protein mRNA. It should be pointed out that this experiment could not deter- 


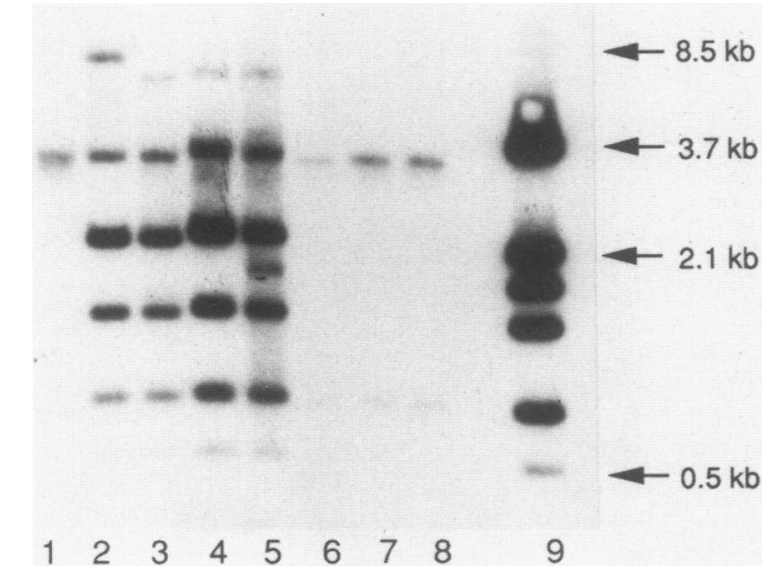

FIG. 3. Hybridization of A gene sequences to DNAs isolated before autogamy from $\mathrm{d} 48$ cell lines injected with pSA8.8R. Total genomic DNAs were digested with HindIII, electrophoresed on $0.8 \%$ agarose, blotted to nitrocellulose, and hybridized with labeled pSA8.8R. Size markers are indicated on the right. Lanes: $1, \mathrm{~d} 48 ; 2$, wild type; 3 to 5 , d48 cell lines injected with pSA8.8R that were rescued after autogamy; 6 to $8, \mathrm{~d} 48$ cell lines injected with pSA8.8R that were not rescued after autogamy; 9 , pSA8.8R digested with HindIII.

mine whether pSA8.8R rescued d48 with the same efficiency as pSA10XS, since there may have been transformed cell lines that were not subsequently rescued and therefore went undetected.

Specificity of rescue. Since all of the plasmids used to transform $\mathrm{d} 48$ in previous studies rescued the cell line, we performed several controls to determine whether rescue of the cells is a specific effect and not merely the result of vector sequences or injection of a large mass of DNA. All experiments contained uninjected control cells to measure the background reversion rate. Approximately $3 \%$ of all uninjected cells reverted to the wild type during this study. Calf thymus DNA was microinjected into macronuclei to test the ability of bulk DNA to rescue the $\mathrm{d} 48$ cell line; $9 \%$ of these cell lines reverted to $\mathrm{A}^{+}$after autogamy. If transformation with specific DNA is required for rescue of the d48 cell line, then the frequency of reversion of cells injected with DNA but not transformed should be low. Approximately $13 \%$ of cells injected with pSA10XS but not transformed (phenotypically $\mathrm{A}^{-}$before autogamy) reverted to $\mathrm{A}^{+}$ after autogamy. This frequency is considerably lower than the $100 \%$ for stable transformants but is greater than the $3 \%$ background reversion rate and could be the result of a low copy number of the A gene in some cells, insufficient to express the A gene but enough to induce the cytoplasmic effect. To demonstrate the specificity of the A gene DNA sequences further, we sought to identify a smaller fragment of the A locus that was not able to rescue the mutant (alternatively, this might have localized the functional region to a smaller fragment). A 2.1-kb HindIII fragment located in the middle of the A gene coding region was chosen. DNAs were isolated from all injected cell lines before autogamy and scored for transformation by Southern hybridization; after autogamy, all cell lines were scored for A expression with anti-A serum and a subset was also scored by Southern hybridization. The Southern blot shown in Fig. 4 shows a representative sample of DNAs isolated before autogamy from injected cell lines. Lanes 2 to 8 in Fig. 4 contained

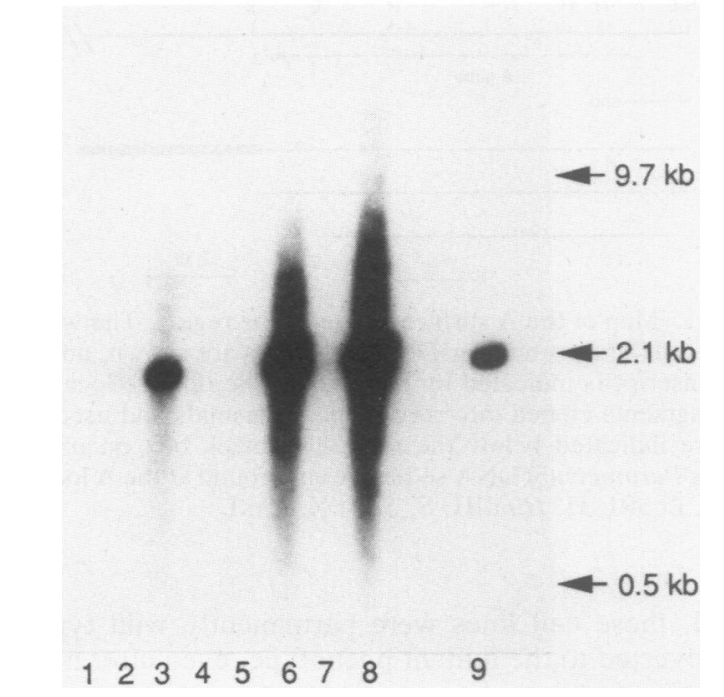

FIG. 4. Hybridization of DNAs isolated before autogamy from d48 cell lines injected with pSA2.1H. Total genomic DNAs were digested with HindIII, electrophoresed on $0.8 \%$ agarose, blotted to nitrocellulose, and hybridized with nick-translated $\mathrm{pSA2.1H}$. Lanes: $1, \mathrm{~d} 48 ; 2$ to $8, \mathrm{~d} 48$ cell lines postinjection of pSA2.1H but before autogamy; 9 , pSA2.1H digested with HindIII. Size markers are indicated on the right.

DNAs from cell lines injected with pSA2.1H; hybridization with the 2.1-kb HindIII fragment indicated that lanes 3, 6, and 8 contained DNAs from transformed cell lines. None of the cell lines injected with pSA2.1H were rescued after autogamy, despite transformation of 20 independent cell lines. These data demonstrate that rescue of $\mathrm{d} 48$ was the result of transformation with specific Paramecium DNA sequences.

\section{DISCUSSION}

We have shown in this report that macronuclear transformation of $\mathrm{d} 48$ cells with portions of the A gene locus is sufficient to rescue the mutant line. It is unlikely that rescue of $\mathrm{d} 48$ is the result of micronuclear transformation. The Mendelian $\mathrm{A}^{-}$mutant $\mathrm{d} 12$ has been repeatedly transformed with $\mathrm{A}$ gene plasmids but reverts to $\mathrm{A}^{-}$after autogamy, as expected for a macronuclear transformant (6). In addition, a recent series of elegant micronuclear transfer experiments by Kobayashi and Koizumi has shown that wild-type and d48 micronuclei are functionally equivalent (9); therefore, the inability of $\mathrm{d} 48$ cells to include the $A$ gene in the macronucleus is entirely the result of a macronuclear mutation. Three observations indicate that the effect of the mutation in $\mathrm{d} 48$ is specific for the A locus. (i) Other genetic markers are unaffected by $\mathrm{d} 48$ cytoplasm in genetic crosses (3). (ii) Five randomly selected telomere DNA clones and a middle repetitive DNA sequence hybridize to identical-size DNA fragments in wild-type and d48 cells (5). (iii) The d48 cell line is healthy and has no pleiotropic effects, suggesting that no essential genes are affected.

By injecting a fragment of the A gene that is unable to express the A surface protein, we have shown that rescue is not dependent on the complete A gene product. Although it is possible that the sequences are passive and do not actively produce a product (for example, the DNA sequences bind a negative factor), this seems unlikely, since results of genetic 
studies and cytoplasm microinjection $(3,8)$ strongly support a model in which wild-type cytoplasm actively provides a component missing in d48. The molecular explanation for the ability of the macronuclear A gene sequences to influence DNA processing is unknown. Any model must account for several observations. (i) The old macronucleus produces a cytoplasmic factor that interacts with the developing macronucleus. (ii) Loss of the factor results in a very specific defect in the macronucleus. (iii) The factor is present (or active) only in cells undergoing sexual reproduction. A speculative model to explain these observations is as follows. A product that may be unrelated to the A surface protein is made from the 8.8-kb EcoRI fragment in the macronucleus of a wild-type cell. This product is made during development of a new macronucleus and is then transported to the cytoplasm, where it interacts with the macronuclear anlagen and is required for correct processing of the A gene. In the d48 macronucleus, there is no $8.8-\mathrm{kb}$ EcoRI fragment; therefore, the product cannot be made and the next macronucleus is defective. Since the entire A gene is not necessary to rescue the cell line, we have considered the possibility that other gene products are made from these DNA sequences. The complete sequence of the $8.8-\mathrm{kb}$ EcoRI fragment has been determined and analyzed for alternative open reading frames in both orientations (12). A number of alternative open reading frames were identified and compared with the available DNA and protein data bases (GenBank and EMBL), but no significant similarities were found. We should add that on the basis of current experimental data, there is no reason to assume that the cytoplasmic factor is a protein rather than an RNA molecule.

Our model for the d48 cell line also has implications for other traits with similar inheritance. Both mating type and a mutation that affects secretory organelle ontogeny in $P$. tetraurelia are traits determined by the macronucleus and inherited according to the cytoplasm of the parental cell (17). More recently, non-Mendelian mutations affecting the B surface protein locus have been isolated (13). We propose that each of these traits is also controlled by the presence or absence of specific DNA sequences in the macronucleus and that the cytoplasmic state is also controlled by the presence of that DNA sequence.

It is interesting that in most examples of non-Mendelian inheritance in paramecia the phenotype and cytoplasmic type are linked, just as the $A^{-}$phenotype is linked in $\mathrm{d} 48$ to the mutant cytoplasmic state. An interesting exception to this rule are homozygotes for the allele $\mathrm{mt}^{\circ}$ in $P$. septaurelia. These cells are always mating type $O$ but possess the $E$ cytoplasmic state; thus, the phenotype is uncoupled from the cytoplasmic type (19). Similarly, we have shown that the $\mathrm{A}^{-}$ phenotype can be uncoupled from the cytoplasmic type in $\mathrm{d} 48$ by injection of the 8.8-kb EcoRI fragment that generates wild-type cytoplasm yet is incapable of $A$ type surface protein expression.

The possibility that cytoplasmic control of macronuclear DNA content is a general feature of macronuclear development remains. The relatively small number of non-Mendelian traits observed thus far in paramecia is expected, since any gene essential for viability presumably cannot be in this category (a macronuclear deletion would be lethal). There is evidence that other ciliated protozoa contain cytoplasmic determinants that affect macronuclear development. A class of mutations that act through the cytoplasm during macronuclear development and affect surface protein gene expression in Tetrahymena thermophila has been described (2).
Unfortunately, the DNA sequences affected by this cytoplasmic determinant have not been isolated, so molecular analysis is not possible. Our work is now focused on identifying the molecular nature of the cytoplasmic factor that affects the $\mathbf{A}$ gene sequences and its mode of action. A molecular explanation of the inheritance of the $\mathrm{d} 48$ mutation will significantly increase our understanding of the components involved in processing macronuclear DNA in ciliates and could potentially reveal novel nucleic acid biochemistry.

\section{ACKNOWLEDGMENTS}

This research was supported by Public Health Service grant GM43357 from the National Institutes of Health to J.F. and a Junior Faculty Award from the American Cancer Society to J.F.

\section{REFERENCES}

1. Caron, F., and E. Meyer. 1989. Molecular basis of surface antigen variation in paramecia. Annu. Rev. Microbiol. 43:23-43.

2. Doerder, F. P., and M. S. Berkowitz. 1987. Nucleo-cytoplasmic interaction during macronuclear differentiation in ciliate protists: genetic basis for cytoplasmic control of SerH expression during macronuclear development in Tetrahymena thermophila. Genetics 117:13-23.

3. Epstein, L. M., and J. D. Forney. 1984. Mendelian and nonMendelian mutations affecting surface antigen expression in Paramecium teraurelia. Mol. Cell. Biol. 4:1583-1590.

4. Forney, J. D., L. M. Epstein, L. M. Preer, B. M. Rudman, D. J. Widmayer, W. H. Klein, and J. R. Preer, Jr. 1983. Structure and expression of genes for surface proteins in Paramecium. Mol. Cell. Biol. 3:466-474.

5. Forney, J. D., and S. Fong. Unpublished data.

6. Godiska, R., K. Aufderheide, D. Gilley, P. Hendrie, T. Fitzwater, L. Preer, B. Polisky, and J. Preer, Jr. 1987. Transformation of Paramecium by microinjection of a cloned serotype gene. Proc. Natl. Acad. Sci. USA 84:7590-7594.

7. Harumoto, T. 1986. Induced change in a non-Mendelian determinant by transplantion of macronucleoplasm in Paramecium tetraurelia. Mol. Cell. Biol. 6:3498-3501.

8. Koizumi, S., and S. Kobayashi. 1989. Microinjection of plasmid DNA encoding the A surface antigen of Paramecium tetraurelia restores the ability to regenerate a wild-type macronucleus. Mol. Cell. Biol. 9:4398-4401.

9. Koizumi, S., and S. Kobayashi. Personal communication.

10. Maniatis, T., E. F. Fritsch, and J. Sambrook. 1982. Molecular cloning: a laboratory manual. Cold Spring Harbor Laboratory, Cold Spring Harbor, N.Y.

11. Martin, L., H. Murray, and J. R. Preer. Personal communication.

12. Nielsen, E., and J. Forney. Unpublished data.

13. Preer, J. R. Personal communication.

14. Preer, J. R., Jr., L. B. Preer, B. Rudman, and A. Barnett. 1987. Molecular biology of the genes for immobilization antigens in Paramecium. J. Protozool. 34:418-423.

15. Sonneborn, T. M. 1950 . Methods in the general biology and genetics of Paramecium aurelia. J. Exp. Zool. 113:87-148.

16. Sonneborn, T. M. 1970 . Methods in paramecium research. Methods Cell Physiol. 4:241-339.

17. Sonneborn, T. M. 1977 . Genetics of cellular differentiation: stable nuclear differentiation in eukaryotic unicells. Annu. Rev. Genet. 11:349-367.

18. Sonneborn, T. M., and M. Schneller. 1979. A genetic system for alternative stable characteristics in genomically identical homozygous clones. Dev. Genet. 1:21-46.

19. Taub, S. R. 1963. The genetic control of mating type differentiation in Paramecium. Genetics 48:815-834.

20. Tondravi, M. M., and M.-C. Yao. 1986. Transformation of Tetrahymena thermophila by microinjection of ribosomal RNA genes. Proc. Natl. Acad. Sci. USA 83:4369-4373.

21. Vieira, J., and J. Messing. 1987. Production of single-stranded plasmid DNA. Methods Enzymol. 153:3-11. 